\title{
Superação de Dormência em Sementes da Liana Abrus precatorius L.
}

\section{Erika Cortines ${ }^{1}$, Luana Almeida Bianquini ${ }^{1}$, Ana Helena de Souza Dias ${ }^{2}$, Joel Quintino de Oliveira Junior ${ }^{2}$, Tiago Böer Breier ${ }^{3}$}

\footnotetext{
${ }^{1}$ Programa de Pós-Graduação em Ciências Ambientais e Florestais, Universidade Federal Rural do Rio de Janeiro - UFRRJ
}

${ }^{2}$ Programa de Pós-Graduação em Engenharia Florestal, Universidade Federal Rural do Rio de Janeiro - UFRRJ

${ }^{3}$ Departamento de Silvicultura, Instituto de Florestas, Universidade Federal Rural do Rio de Janeiro - UFRRJ

\section{RESUMO}

As sementes da liana Abrus precatorius L. (Fabaceae) foram submetidas a diferentes tratamentos para a superação da dormência tegumentar: T1) controle; T2) escarificação mecânica; T3) imersão em água a $80{ }^{\circ} \mathrm{C}$ por 5 minutos; T4) imersão em ácido sulfúrico por 15 minutos; e T5) imersão em ácido sulfúrico por 30 minutos. A escarificação mecânica e imersão em ácido sulfúrico por 30 minutos foram os tratamentos mais eficientes na superação de dormência das sementes de Abrus precatorius.

Palavras-chave: dormência, Abrus precatorius, sementes.

\section{Dormancy Breaking in Seeds of the Liana Abrus precatorius L.}

\begin{abstract}
Liana Abrus precatorius L. (Fabaceae) seeds were submitted to the following different treatments in order to break the tegument dormancy: T1) control; T2) mechanical scarification; T3) immersion in water at $80^{\circ} \mathrm{C}$ for 5 minutes; T4) immersion in sulfuric acid for 15 minutes and T5) immersion in sulfuric acid for 30 minutes. The mechanical scarification treatment and the immersion in sulfuric acid for 30 minutes were the most efficient treatments to break the dormancy of Abrus precatorius seeds.
\end{abstract}

Keywords: dormancy, Abrus precatorius, seeds. 


\section{INTRODUÇÃO}

Algumas espécies vegetais apresentam dormência como uma forma de adaptação evolutiva para que a germinação ocorra em períodos alternados, aumentando a chance de encontrar condições propícias, evitando assim a morte dos indivíduos e possibilitando maior sobrevivência e estabelecimento da espécie nos mais variados ambientes (Vieira \& Fernades, 1997; Fowler \& Bianchetti, 2000; Zaidan \& Barbedo, 2004). A dormência pode ser causada por substâncias inibidoras (dormência química), por resistência mecânica dos tecidos externos ao embrião (mecânica), impermeabilidade do tegumento (física), pela imaturidade do embrião (morfológica) ou por sua dormência (fisiológica) (Kramer \& Kozlowski, 1972; Fowler \& Bianchetti, 2000).

Em diversas espécies, a dormência é considerada um dos problemas mais comuns na conservação de germoplasma e um empecilho para a produtividade em larga escala (Vazquez-Yanes \& OrozcoSegovia, 1993). O desconhecimento da superação de dormência pode subestimar a capacidade de germinação de um lote, revelando valores baixos de porcentagem de germinação.

A espécie Abrus precatorius L. pertence à família Fabaceae, subfamília Faboideae, e apresenta hábito escandente. A espécie é originária da Índia e ocorre de forma espontânea em vários continentes. No Brasil é conhecida popularmente como jequitiri, olho-de-pombo, olho-de-saci, olho-de-flamingo e tento (Olsnes et al., 1975; Liener, 1976). Apresenta substâncias tóxicas nas sementes (Olsnes et al., 1975; Liener, 1976) com efeitos tóxicos estudados em bovinos (Tokarnia \& Döbereiner, 1997).

$\mathrm{Na}$ área da saúde pública, a espécie pode se tornar de grande importância devido a seus efeitos letais para os transmissores da esquistossomose (Sparg et al., 2000) e efeito larvicida sobre a espécie Culex quinquefasciatus Say (Muthukrishnan et al., 1997), além de usos experimentais no combate ao câncer (Reddy \& Sirsi, 1969; Matos, 2008).

Apesar de sua alta toxidade, as sementes de Abrus precatorius quando fervidas são utilizadas para consumo humano na Índia, servindo como fonte de proteínas para a população (Rajaram \& Janardhanan, 1992).
No Brasil, as sementes são também usadas por suas características ornamentais, sendo utilizada em artesanatos pelas populações tradicionais, principalmente na confecção de bijuterias, por apresentarem tegumento resistente com as cores vermelho e preto (Bandeira \& Pagano, 2008; Valle, 2008).

No entanto, a ecologia da espécie ainda é pouco conhecida, com destaque para os trabalhos de fenologia em Santa Catarina (Mafra \& Araújo, 2004) e de interações entre espécies, no qual Veenakumari et al. (1997) encontraram associação de Abrus precatorius com larvas de borboletas que utilizam a flor como alimento e como hospedeira, em Ilhas no Oceano Índico.

Entender os processos de superação de dormência das sementes de Abrus precatorius pode se tornar uma forma de viabilizar a produção de mudas da espécie para fins de geração de renda. $\mathrm{O}$ presente estudo teve por objetivo avaliar o efeito de tratamentos para superação de dormência na germinação de sementes de Abrus precatorius.

\section{MATERIAL E MÉTODOS}

O experimento foi conduzido no Laboratório de Biologia Reprodutiva e Conservação de Espécies Arbóreas (LACON) da Universidade Federal Rural do Rio de Janeiro (UFRRJ). As sementes foram coletadas no campus da UFRRJ nas proximidades do Instituto de Florestas, sendo o procedimento de colheita e beneficiamento feitos manualmente. Seguindo método proposto pelas Regras de Análise de Sementes (Brasil, 2009), foi determinado o teor de umidade das sementes e peso de 1000 sementes.

Os tratamentos utilizados para testar a superação de dormência foram: T1) controle, no qual as sementes foram colocadas para germinar sem nenhum tratamento; T2) escarificação mecânica; T3) imersão em água a $80{ }^{\circ} \mathrm{C}$ por 5 minutos; T4) imersão das sementes em ácido sulfúrico $\left(\mathrm{H}_{2} \mathrm{SO}_{4}\right)$ por 15 minutos; e T5) imersão das sementes em ácido sulfúrico $\left(\mathrm{H}_{2} \mathrm{SO}_{4}\right)$ por 30 minutos. A escarificação mecânica foi cuidadosamente realizada utilizando um esmeril, sendo que as sementes foram encostadas no disco do esmeril e submetidas à abrasão no lado 
oposto ao embrião. Para cada tratamento, foram usadas oito repetições de 25 sementes.

Uma semana após a colheita, as sementes foram colocadas para germinar sobre vermiculita em caixas acrílicas do tipo gerbox, dispostas em câmara de germinação com luz constante à temperatura de $30{ }^{\circ} \mathrm{C}$. As contagens foram feitas ao final de cada semana, durante cinco semanas consecutivas, sendo avaliados os seguintes parâmetros: número de sementes duras, número de sementes embebidas, número de sementes emitindo radícula, número de plântulas normais e percentual de germinação. Foram consideradas plântulas normais aquelas com o primeiro par de folíolos evidentes.

Na comparação estatística entre os tratamentos foi utilizado o teste Kruskal-Wallis, devido às variâncias serem desiguais, com nível de decisão de $5 \%$.

\section{RESULTADOS E DISCUSSÃO}

Para as sementes de Abrus precatorius estudadas, o percentual de umidade encontrado após a coleta e beneficiamento foi de $15,9 \%$ e o peso de 1000 sementes, de $104,1 \mathrm{~g}$.

A Tabela 1 apresenta os resultados em percentuais dos parâmetros analisados para os cinco tratamentos. Considerando o parâmetro percentual de germinação, os tratamentos escarificação mecânica e imersão em ácido sulfúrico por 30 minutos foram os mais eficientes na superação de dormência das sementes de Abrus precatorius, com 88 e 52\% de sementes germinadas, respectivamente, conforme a Figura 1. Contudo, o tratamento imersão em ácido sulfúrico por 30 minutos, se encontra em situação

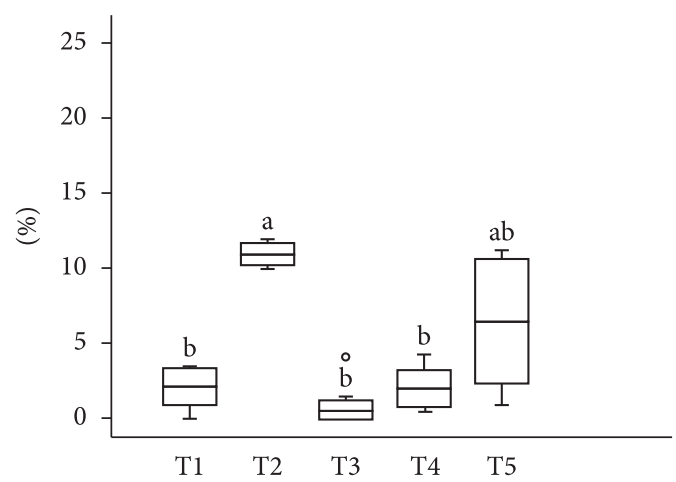

Figura 1. Percentual de plântulas normais de Abrus precatorius para diferentes tratamentos de superação de dormência: T1) controle; T2) escarificação mecânica; T3) água a $80^{\circ} \mathrm{C}$ por 5 minutos; T4) ácido sulfúrico por 15 minutos; e T5) ácido sulfúrico por 30 minutos. Nas caixas estão indicados média, desvio padrão e valores máximo e mínimo para oito repetições por tratamento. Letras diferentes indicam diferença estatística $(\mathrm{p}<0,05)$. Figure 1. Percentage of normal seedlings of Abrus precatorius for different treatments to break the tegument dormancy: T1) control, T2) mechanical scarification; T3) water at $80{ }^{\circ} \mathrm{C}$ for 5 minutes, T4) sulfuric acid for 15 minutes, and T5) sulfuric acid for 30 minutes. Boxes are given mean, standard deviation and maximum values and minimum values for eight replicates. Letters indicate statistically different $(\mathrm{p}<0.05)$.

Tabela 1. Valores em percentual para sementes de Abrus precatorius em diferentes tratamentos para a superação de dormência: T1) controle; T2) escarificação mecânica; T3) água a $80{ }^{\circ} \mathrm{C}$ por 5 minutos; T4) ácido sulfúrico por 15 minutos; e T5) ácido sulfúrico por 30 minutos. Valores referentes à data de encerramento do teste para sementes duras, sementes embebidas e emitindo radícula. Valores cumulativos para semente morta, plântula anormal e plântula normal.

Table 1. Figures in percentage for seeds of Abrus precatorius in different treatments to break the tegument dormancy: T1) control, T2) mechanical scarification; T3) water at $80{ }^{\circ} \mathrm{C}$ for 5 minutes, T4) sulfuric acid for 15 minutes, and T5) sulfuric acid for 30 minutes. Data to the closing date of the test for seed hard, imbibed seeds and radicles emission. Cumulative data for dead seed, abnormal and normal seedling.

\begin{tabular}{lrrrrr}
\multicolumn{1}{c}{ Parâmetros } & \multicolumn{5}{c}{ Tratamentos } \\
\cline { 2 - 6 } \multicolumn{1}{c}{ T1 } & T2 & T3 & T4 & T5 \\
\hline Sementes duras & 73,0 & 0,0 & 35,7 & 18,2 & 0,5 \\
Sementes embebidas & 2,5 & 3,5 & 44,9 & 25,8 & 12,3 \\
Sementes emitindo radícula & 6,4 & 0,0 & 2,0 & 36,8 & 29,9 \\
Sementes mortas & 0,5 & 7,5 & 9,2 & 2,9 & 5,4 \\
Plântulas anormais & 0,5 & 1,0 & 0,0 & 0,0 & 0,0 \\
Plântulas normais & 17,2 & 88,0 & 8,2 & 16,3 & 52,0 \\
\hline
\end{tabular}


intermediária, participando estatisticamente ( $p>0,05)$ do grupo dos tratamentos mais eficientes na superação de dormência e simultaneamente do grupo dos tratamentos com menor eficiência na superação de dormência (Figura 1).

A eficiência da superação de dormência também pode ser avaliada através do parâmetro percentual de sementes duras, em que os tratamentos escarificação mecânica, imersão em ácido sulfúrico por 30 minutos e imersão em ácido sulfúrico por 15 minutos não diferem estatisticamente entre si ( $\mathrm{p}>0,05$ ), com percentuais que variam de 0 a 18,2\% de sementes duras, sendo assim são os tratamentos mais eficientes para a superação de dormência conforme a Figura 2. Outro grupo foi formado pelos tratamentos com grande quantidade de sementes duras, no qual novamente participa o tratamento imersão em ácido sulfúrico por 15 minutos (18,2\%), os tratamentos água quente $(35,7 \%)$ e controle (73,0\%). O tratamento imersão em ácido sulfúrico

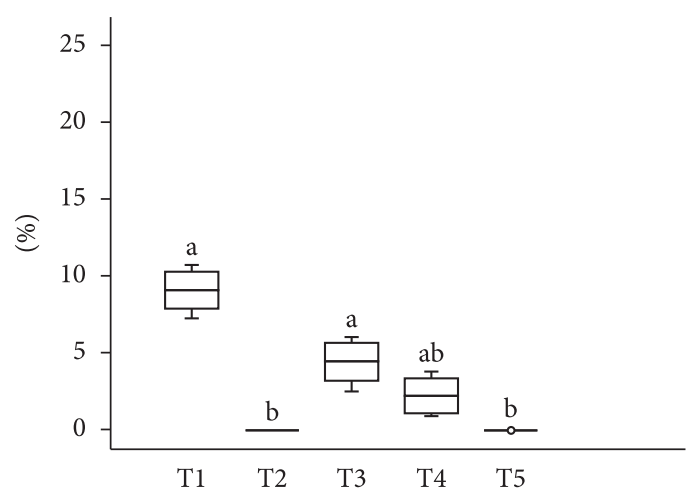

Figura 2. Percentual de sementes duras de Abrus precatorius para diferentes tratamentos de superação de dormência: T1) controle; T2) escarificação mecânica; T3) água a $80^{\circ} \mathrm{C}$ por 5 minutos; T4) ácido sulfúrico por 15 minutos; e T5) ácido sulfúrico por 30 minutos. Nas caixas estão indicados média, desvio padrão e valores máximo e mínimo para oito repetições por tratamento. Letras diferentes indicam diferença estatística $(\mathrm{p}<0,05)$. Figure 2. Percentage of hard seeds of Abrus precatorius for different treatments to break tegument dormancy: T1) control, T2) mechanical scarification; T3) water at $80{ }^{\circ} \mathrm{C}$ for 5 minutes, T4) sulfuric acid for 15 minutes, and T5) sulfuric acid for 30 minutes. In boxes are shown average values and standard deviation maximum and minimum values for eight replicates.Different letters indicate statistical difference $(\mathrm{p}<0.05)$. por 15 minutos participa dos dois grupos devido aos valores intermediários do percentual de sementes duras obtidos.

O tratamento com escarificação mecânica foi o único que apresentou o pico de sementes embebidas já na primeira semana de avaliação (Figura 3). Este padrão não foi observado nos outros tratamentos, sendo que para eles o pico de sementes embebidas foi entre a segunda e quarta semana. Considerando

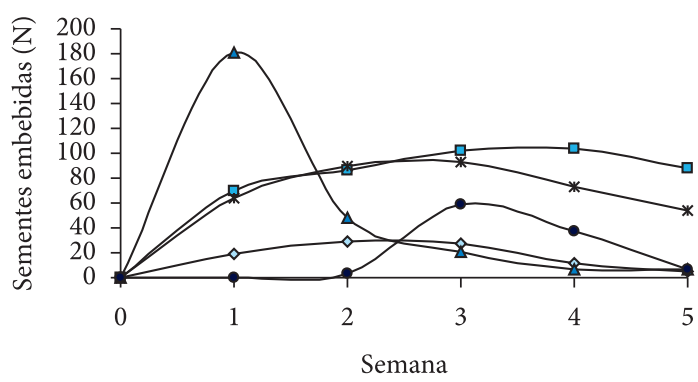

$$
\begin{aligned}
& \diamond \text { Controle } \quad-\text { - Água } 5 \text { min } \\
& - \text { Escarificação } \quad \text { * Ác. Sulf. } 15 \text { min } \\
& \rightarrow \text { Ác. Sulf. } 30 \mathrm{~min}
\end{aligned}
$$

Figura 3. Número de sementes embebidas de Abrus precatorius ao longo de cinco semanas de avaliação para diferentes tratamentos de superação de dormência.

Figure 3. Number of imbibed seeds of Abrus precatorius over five weeks of evaluation for different treatments for break tegument dormancy.

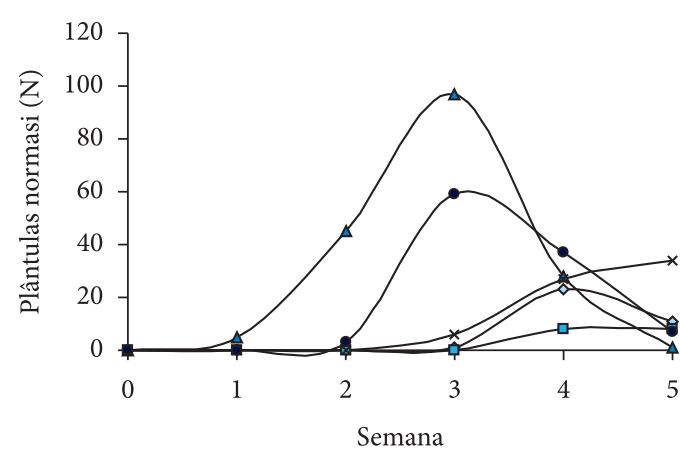

$$
\begin{aligned}
& \prec \text { Água } 5 \text { min } \quad-\square-\text { Controle } \\
& ₫ \text { Escarificação } \quad * \text { Ác. Sulf. } 15 \text { min } \\
& \multimap \text { Ác. Sulf. } 30 \text { min }
\end{aligned}
$$

Figura 4. Número de plântulas normais de Abrus precatorius ao longo de cinco semanas de avaliação para diferentes tratamentos de superação de dormência.

Figure 4. Number of normal seedlings of Abrus precatorius over five weeks of evaluation for different treatments for break tegument dormancy. 
o número de plantas normais (Figura 4), os tratamentos escarificação mecânica e ácido sulfúrico por 30 minutos apresentam pico na terceira semana de avaliação. Estes resultados mostram que a escarificação mecânica foi mais eficaz na superação da dormência das sementes de Abrus precatorius.

Em testes de laboratório, Rizzini (1976) relatou a presença de dormência tegumentar para Abrus precatorius. Outros estudos citam que em 260 espécies de leguminosas avaliadas, 85\% apresentaram sementes com tegumento total ou parcialmente impermeável à água (Rolston, 1978), mostrando que nesta família este tipo de dormência é comum (Villiers, 1972; Mayer \& Poljakoff-Mayber, 1989).

Os resultados do presente trabalho corroboram com estudos realizados com outras espécies de leguminosas arbóreas como Bowdichia virgilioides Kunth., Caesalpinia leiostachya (Benth.) Ducke e Cassia javanica Ried., nos quais o melhor tratamento para a superação da dormência tegumentar foi a escarificação mecânica (Grus et al., 1984; Smiderle \& Souza, 2003).

A falta de efeito observado no tratamento com água quente indica a necessidade de novos testes com aumento do tempo de exposição (5 minutos) das sementes à alta temperatura $\left(80^{\circ} \mathrm{C}\right)$ e/ou aumento da temperatura para elucidar esta questão. A escolha deste tratamento (água a $80{ }^{\circ} \mathrm{C}$ por 5 minutos) foi baseada em estudos realizados com a leguminosa arbórea Copaifera langsdorffii Desf. (Borges et al., 1982).

Tratamentos para superação de dormência com ácido sulfúrico apresentam praticidade principalmente para sementes de tamanho diminuto como as sementes de Abrus precatorius. Outras espécies de leguminosas como Senna macranthera (DC. ex Collad.) H.S. Irwin \& Barneby e Piptadenia moniliformis Benth. apresentaram bons resultados para superação de dormência em tratamentos com ácido sulfúrico (Santarém \& Aquila, 1995; Azeredo et al., 2010). Recomendamos que em futuros estudos com sementes de Abrus precatorius sejam testados tempos de exposição ao ácido sulfúrico superiores a 30 minutos.

\section{CONCLUSÕES}

A escarificação mecânica e imersão em ácido sulfúrico por 30 minutos foram os tratamentos mais eficientes na superação de dormência tegumentar de Abrus precatorius. $\mathrm{O}$ conhecimento sobre métodos para a superação da dormência de sementes contribui para o aumento da eficiência da germinação no processo produtivo de mudas, além de proporcionar maior uniformidade na germinação.

\section{AGRADECIMENTOS}

Agradecemos à Bióloga Fabiana Umetsu as sugestões ao manuscrito.

\section{STATUS DA SUBMISSÃO}

Recebido: 21/09/2009

\section{AUTOR(ES) PARA CORRESPONDÊNCIA}

\section{Tiago Böer Breier}

Departamento de Silvicultura,

Instituto de Florestas,

Universidade Federal Rural do Rio de Janeiro -

UFRRJ, Rod. Br 465, Km 7, s/n, CEP 23890-000,

Seropédica, RJ, Brasil

e-mail: tiagobreier@gmail.com

\section{REFERÊNCIAS}

Azeredo GA, De Paula RC, Valeri SV, Moro FV. Superação de dormência de Piptadenia moniliformis Benth. Revista Brasileira e Sementes 2010, 32(2):49-58. doi:10.1590/S0101-31222010000200006

Bandeira J, Pagano S. Sementes ornamentais no Brasil. Rio de Janeiro: Reler; 2008.

Borges EEL, Borges RCG, Candido JF, Gomes JM. Comparação de métodos de quebra de dormência em sementes de copaíba. Revista Brasileira de Sementes 1982; 04(1):09-12.

Brasil. Regras para análise de sementes. Brasília: MAPA/ ACS; 2009.

Fowler JAP, Bianchetti A. Dormência em sementes florestais. Colombo: EMBRAPA-Florestas; 2000.

Grus VM, Demattë MESP, Graziano TT. Germinação de sementes de pau-ferro e cássia-javanesa submetidas 
a tratamentos para quebra de dormência. Revista Brasileira de Sementes 1984; 06(02):29-36.

Kramer PJ, Kozlowski T. Fisiologia das árvores. Lisboa: Fundação Calouste Gulbenkian; 1972.

Liener IE. Phytohemagglutinins (Phytolectins). Annual Review Plant Physiology 1976; 27:291-319. doi:10.1146/ annurev.pp.27.060176.001451

Mafra AI, Araujo AC. Fenologia de Abrus precatorius L. (Fabaceae) em Navegantes, Santa Catarina, Brasil. In: Anais do I Congresso Nacional de Oceanografia; 2004; Itajaí. Itajaí: Univali; 2004.

Matos DC. Estudo comparativo da atividade antiinflamatória e anti-tumoral de proteinas inativadoras de ribossomos extraídas de plantas [dissertação]. Araraquara: Universidade do Estado de São Paulo UNESP, 2008.

Mayer AM, Poljakoff-Mayber A. The germination of seeds. Oxford: Pergamon Press; 1989.

Muthukrishnan J, Pushpalatha E, Kasthuribai A. Biological effects of four plant extracts on Culex quinquefasciatus Say larval stages. Insect Science and its Applications 1997; 17:389-394.

Olsnes S, Refsnes K, Christensen TB, Pihl A. Studies on the structure and properties of the lectins from Abrus precatorius and Ricinus communis. Biochimica et Biophysica Acta 1975; 405(1):1-10.

Rajaram N, Janardhanan K. The chemical composition and nutritional potential of the tribal pulse, Abrus precatorius L. Plant Foods for Human Nutrition 1992; 4:285-290. doi:10.1007/BF02194088

Reddy VV, Sirsi M. Effect of Abrus precatorius L. on experimental tumors. Cancer Research 1969, 29:1447-1451.

Rizzini CT. Tratado de Fitogeografia do Brasil: aspectos ecológicos. São Paulo: EDUSP; 1976.

Rolston MP. Water impermeable seed dormancy. The Botanical Review 1978; 44:365-396. doi:10.1007/ BF02957854

Santarém ER, Áquila MEA. Influência de métodos de superação de dormência e do armazenamento na germinação de sementes de Senna macranthera (Colladon) Irwin \& Barneby (Leguminosae). Revista Brasileira de Sementes 1995; 17(2):205-209.

Smiderle IG, Sousa GD. Dormência em sementes de paricarana (Bowdichia virgilioides Kunth - Fabaceae - Papilionidae). Revista Brasileira de Sementes 2003; 25(2):48-52. doi:10.1590/S0101-31222003000400007

Sparg SG, Van Staden J, Jäger AK. Efficiency of traditionally used South African plants against Schistosomiasis. Journal of Ethnopharmacology 2000; 73:209-214

Tokarnia $\mathrm{CH}$, Döbereiner J. Imunidade cruzada pelas sementes de Abrus precatorius e Ricinus communis em bovinos. Pesquisa Veterinária Brasileira 1997; 17(1):25-35. doi:10.1590/S0100-736X1997000100005

Valle MJLV. Sementes florestais utilizadas em artesanato no Rio de Janeiro [monografia]. Seropédica: Instituto de Florestas, Universidade Federal Rural do Rio de Janeiro; 2008.

Vazquez-Yanes C, Orozco-Segovia A. Patterns of seed longevity and germination in the tropical rainforest. Annual Review of Ecology and Systematics 1993; 24:69-87. doi:10.1146/annurev.es.24.110193.000441

Veenakumari K, Mohanraj P, Sreekumar PV. Host plant utilization by butterfly larvae in the Andaman and Nicobar Islands (Indian Ocean). Journal of Insect Conservation 1997; 1:235-246. doi:10.1023/A:1018424302583

Vieira IG, Fernades GD. Métodos de quebra de dormência de sementes Piracicaba: IPEFLCF/ESALQ/ USP; 1997. [cited 2009 jul. 10]. Informativo Sementes IPEF. Available from: http://www.ipef.gov.br.

Villiers TA. Seed dormancy. In: Kozlowsky TT., ed. Seed biology. New York: Academic Press; 1972. v. 2, p. 220-282.

Zaidan LBP, Barbedo CJ. Quebra de dormência em sementes. In: Ferreira AG, Borghetti F, org. Germinação: do básico ao aplicado. Porto Alegre: Artmed; 2004. p. 135-146. 